\title{
SOCIOLOGÍA DEL CONOCIMIENTO, TEORÍA DE LOS CAMPOS Y BIBLIOMETRÍA
}

\author{
Luis Ernesto Paz Enrique* \\ https://orcid.org/0000-0001-9214-3057 \\ Gloria Ponjuán Dante** \\ https://orcid.org/0000-0003-2063-0934
}

RECIBIDO: Mayo 2021 / ACEPTADO: Noviembre 2021 / PUBLICADO: Enero 2022

Como citar: Paz Enrique, Luis; Ponjuán Duarte, Gloria. (2022). Sociología del conocimiento, teoría de los campos y bibliometría. Telos: revista de Estudios Interdisciplinarios en Ciencias Sociales, 24 (1), Venezuela. (Pp. 157-175).

DOI: www.doi.org/10.36390/telos241.10

\section{RESUMEN}

La Teoría de los Campos es una de las perspectivas de investigación más empleadas desde el ámbito sociológico. Esta concepción presenta una especificación dentro de la sociología del conocimiento: la Teoría del Campo Científico. Dada su adaptabilidad a diversos campos, dominios y disciplinas científicas; esta teoría puede ser aplicada a cualquier área del conocimiento. Aunque la efectividad de la bibliometría ha sido ampliamente demostrada en el estudio de temáticas, disciplinas y dominios del conocimiento, se evidencia que ha sido poco utilizada para describir campos científicos. El presente estudio está enfocado en establecer los nexos entre sociología del conocimiento, Teoría de los Campos y bibliometría para describir campos científicos. La investigación clasificó como descriptiva con aporte teórico. Los resultados fueron obtenidos fundamentalmente a través de métodos con orientación teórica y empírica. A nivel empírico fue determinante el análisis documental, utilizando como principal autor a Peirre Bourdieu (1976). En el estudio se identificaron las características de la Teoría del Campo Científico y de la bibliometría. Lo anterior permitió identificar los nodos de intercepción entre ambas concepciones, permitiendo visualizar su alcance en la delimitación y caracterización de la estructura de campos particulares. Se concluyó que el análisis de campos científicos demanda analizar grandes volúmenes de información. La bibliometría como método y disciplina bibliométrica puede favorecer la delimitación de un campo científico desde las relaciones objetivas (publicaciones) entre los agentes del campo. Las variables principales que permiten describir los campos científicos son las relacionadas con la producción, la colaboración y el impacto.

\footnotetext{
* Licenciado en Ciencias de la Información. Estudiante de postgrado de la Universidad Nacional Autónoma de México. Correo electrónico: luisernestopazenrique@gmail.com

** Doctora en Documentación e Información Científica. Profesora Titular. Departamento de Ciencias de la Información. Universidad de La Habana, Cuba. Correo electrónico: ponjuandg@infomed.sld.cu
} 
Palabras claves: Pierre Bourdieu, Teoría de los Campos, Sociología del conocimiento, Bibliometría, Campos científicos.

\title{
Sociology of knowledge, field theory and bibliometrics
}

\begin{abstract}
The Theory of the Fields is one of the most widely used research perspectives from the sociological field. This conception presents a specification within the sociology of knowledge: the Theory of the Scientific Field. Given its adaptability to various fields, domains and scientific disciplines; this theory can be applied to any area of knowledge. Although the effectiveness of bibliometrics has been widely demonstrated in the study of topics, disciplines and domains of knowledge, it is evident that it has been little used to describe scientific fields. The present study is focused on establishing the links between sociology of knowledge, Field Theory and bibliometrics to describe scientific fields. The research classified as descriptive with theoretical contribution. The results were obtained mainly through methods in the theoretical planes. At the empirical level, the documentary analysis was decisive, using Peirre Bourdieu (1976) as the main author. The study identified the characteristics of the Scientific Field Theory and bibliometrics. The above will identify the interception nodes between both conceptions, allowing to visualize their scope in the delimitation and characterization of the structure of particular fields. It was concluded that the analysis of scientific fields requires to processing of large volumes of information. Bibliometry as a bibliometric method and discipline can favor the delimitation of a scientific field from the objective relationships (publications) between the agents of the field. The main variables that allow describing scientific fields are those related to production, collaboration and impact.
\end{abstract}

Keywords: Pierre Bourdieu; Theory of fields; Sociology of knowledge, Bibliometrics; Scientific fields.

\section{Introducción}

La sociología del conocimiento permite decontruir las formas en que se produce, socializa y comunica el conocimiento científico. Esta disciplina abarca los procesos internos y externos que ocurren en el pensamiento para la construcción del conocimiento. La disciplina sociológica propone una metodología aplicable a la institucionalización del conocimiento científico, a los procesos intelectuales de construcción del conocimiento (sociología del conocimiento internalista y externalista), las comunidades científicas y a la actividad y producción intelectual. Esta sociología a criterio de Paz, Núñez y Garcés (2019), se orienta al análisis de las relaciones contexto - sociedad y la situación histórico - concreta como guía de la actividad intelectual y a los procesos de obtención del conocimiento científico.

Consecuentemente, se evidencia la necesidad de establecer los condicionamientos sociales y científicos que construyen las tendencias y modelos teóricos de los campos científicos. El resultado del análisis y delimitación de la estructura de los mismos tiene un carácter interdisciplinar. En consecuencia, la sociología del conocimiento facilita visualizar las tendencias y perspectivas en la caracterización de las construcciones discursivas en la ciencia, que son reflejo de los campos particulares. 
La Teoría de los Campos de Pierre Bourdieu permite analizar la producción de conocimientos desde una perspectiva reflexiva. La sociología del conocimiento ha tenido como limitación el análisis de grandes volúmenes de información. La problemática mencionada es recogida por Bourdieu (2004), al identificar que la producción intelectual de los campos particulares es mayor a la que los investigadores pueden acceder, leer, consultar y procesar. En correspondencia Rodríguez (2017), sugiere el empleo de la bibliometría aunque no establece cuáles indicadores emplear sus implicaciones a la Teoría del Campo Científico de Bourdieu. Sobre lo anterior destaca el antecedente Homo academicus de Bourdieu (2008) donde se muestra el empleo de procederes cuantitativos aplicados a la actividad y producción científica en el ámbito académico.

Para la obtención de este tipo de resultados es necesario el diseño u obtención de un base de datos bibliográfica con la finalidad de procesar grandes volúmenes de información a partir de los metadatos establecidos como parte de la comunicación científica escrita. La producción intelectual es reflejo del grado de consolidación de la comunidad científica y de los agentes. Dentro de la estructura de los campos, se evidencia las prácticas de distribución, construcción, regulación y legitimidad. Al interior se encuentran las posiciones de poder y su distribución, pero a criterio de Alfaraz (2004): "es también entender el posible devenir de la estructura, ya que las propiedades son a la vez objetos deseados que ponen en marcha estrategias de reformulación y apropiación" (p. 223).

Najman y Hewitt (2003), desarrollaron una experiencia en la delimitación de la estructura de un campo a partir de su producción científica e intelectual publicada. La investigación mencionada se muestran las relaciones entre publicaciones y agentes que son núcleo principal de la estructura. El estudio del sociólogo Rodríguez (2017), constituye un análisis de un campo científico a partir del uso del método bibliométrico. La unidad de observación fueron las publicaciones de autores mexicanos sobre comunicación política en la base de datos SciELO. A criterio del autor:

la estrategia metodológica se presenta (...) a través de dos acercamientos, el cuantitativo desde el análisis de las publicaciones y la bibliometría, la cual permite adentrarse a la manera en que se objetivan las relaciones entre los investigadores y las características del campo por medio de sus publicaciones (p. 18).

Griñan y Muñoz (2017a, 2017b), realizaron un análisis de la estructura del campo de los estudios sociales del trabajo, demostrando su existencia, evolución y desarrollo desde 1960 hasta el presente siglo. La investigación de Herrera (2020), destaca por analizar la constitución del campo científico de los estudios sociales rurales desde las producciones científicas. Se realizó un exhaustivo análisis de contenido desde la confección de una base de datos bibliográfica con una gran cantidad de fuentes de información. Los resultados obtenidos son muestra de las relaciones entre las producciones intelectuales y la conformación de un campo.

En los estudios mencionados se destaca la necesidad de deconstruir los saberes y analizar la práctica científica de grupos y comunidades académicas. El tipo de investigación clasifica como analítico - descriptiva, profundiza en las dimensiones de la estructura del campo científico, al establecer relaciones entre las valoraciones de los sujetos y la producción cientííca. Dentro de la sociología del conocimiento se identifican dos enfoques para el análisis: 1), el que estudia los productos de la mente basado en las concepciones e ideas que anteceden dicha 
producción: internalismo y 2), el que favorece el análisis del contexto donde se integra la producción de conocimientos (King, 2000). "Ambas posturas permiten estudiar la ciencia desde un prisma social, sin embargo, una posición intermedia entre ellas proporciona una mejor comprensión de su desarrollo socio - histórico y teórico - metodológico" (Griñan y Muñoz, 2017a, p. 227). Siguiendo esta línea de pensamiento y coincidiendo con Herrera (2020), para el logro de resultados más objetivos deben tomarse una posición que integre el internalismo y el externalismo como posición intermedia, permitiendo visualizar la construcción intelectual dependiente de factores extracientíficos y aquellos internos propios de la ciencia.

La aplicación de técnicas y procederes que permitan la identificación de condicionantes en la producción de conocimientos contribuye a una sociología más objetiva. En la actualidad el conocimiento es generalmente socializado en revistas científicas, las mismas han sido catalogadas como el medio de socialización por excelencia, por lo que el estudio de estos medios y sus comunicaciones se sitúan como centro de la disciplina sociológica mencionada. A partir del posicionamiento anterior se plantea la necesidad de la perspectiva reflexiva de Bourdieu (2003) como vía para solucionar las limitantes de la sociología del conocimiento internalista y el externalista.

A partir de la concepción de Bourdieu (1990), de que "todo campo, en tanto que producto histórico, engendra el interés que es la condición de su funcionamiento" (p. 88), el presente estudio se centra en establecer los nexos entre sociología del conocimiento, Teoría de los Campos y bibliometría para describir campos científicos. Para ello, se observan las relaciones objetivas de los sujetos (las publicaciones) y subjetivas (relaciones entre los sujetos con el campo). Los estudios sociológicos en torno al análisis o la concepción de un campo científico tienden invariablemente a examinar las producciones intelectuales que son muestra de la actividad y las relaciones de los agentes. En consecuencia, se obtiene una amplia producción científica que el investigador analiza para arribar a generalizaciones de las prácticas y estructura del campo.

Una de las formas de cuantificar y visualizar dicha producción es con el empleo del método bibliométrico. Una investigación que solo emplee dicho método conlleva a distorsiones del campo al solo analizar aquellos constructos visibles en la producción objetiva de conocimientos. La perspectiva de la Teoría del Campo Científico de Bourdieu (1976), permite la cualificación de grandes volúmenes de información posibles de procesar con el método bibliométrico que como consecuencia favorece la obtención de una base de datos bibliográfica. El análisis crítico de las producciones discursivas (como parte de las producciones intelectuales) y de los capitales en juego, favorece que se obtenga un reflejo de la realizad con mayor objetividad. Se planteó como objetivo del presente artículo: establecer los nexos entre sociología del conocimiento, Teoría de los Campos y bibliometría para describir campos científicos.

\section{Materiales y métodos}

La investigación clasificó como descriptiva y documental. Presentó aportes teóricos y metodológicos. Se centró en establecer, diseñar y describir los núcleos que desde la bibliometría pueden contribuir a la sociología del conocimiento, especialmente en el análisis de campos cientíicos. La metodología establecida para definir los criterios conceptuales y metodológicos de los campos científicos y su relación con la bibliometría tuvo un enfoque cualitativo. Se emplearon métodos en los planos teórico y empírico para la obtención de resultados. En el 
teórico, se aplicó fundamentalmente el analítico sintético. En el empírico, se empleó el análisis documental.

La técnica que facilitó la recogida de información fue la revisión de documentos. A partir de la misma se identificaron los referentes teóricos propios de un estudio documental. Las fuentes y bases de datos consultadas fueron SciELO, DOAJ y Google Scholar. Las palabras clave definidas que orientaron la búsqueda de recuperación de documentos fueron: sociología, bibliometría, campo científico, Teoría del Campo Científico y evaluación de la ciencia. El período temporal de selección de las fuentes para el estudio documental abarcó desde 1970 hasta la actualidad. Las fuentes con mayor grado de antigüedad permitieron consultar las perspectivas en cuanto a los campos científicos y los colegios invisibles.

Se consultaron 173 documentos que fueron discriminados a partir de criterios de exclusión propuestos para los propósitos del presente estudio. Se definieron categorías que permitieron la extracción de información relevante. En primer lugar, se localizaron los referentes en torno a los campos científicos desde tres disciplinas sociológicas: 1) sociología del conocimiento, 2) sociología de la ciencia y 3) sociología del conocimiento científico. La estrategia de búsqueda y los criterios de exclusión por base de datos se muestra en la Tabla 1.

Tabla 1: estrategia de búsqueda y criterios de exclusión para llevar a cabo la revisión bibliográfica.

\begin{tabular}{|c|c|c|c|c|}
\hline $\begin{array}{l}\text { Base de } \\
\text { datos }\end{array}$ & $\begin{array}{l}\text { Estrategias de } \\
\text { búsqueda }\end{array}$ & $\begin{array}{ll}\text { Criterios } & \text { de } \\
\text { exclusión } & \end{array}$ & $\begin{array}{l}\text { Documentos } \\
\text { recuperados }\end{array}$ & $\begin{array}{l}\text { Documentos } \\
\text { relevantes } \\
\text { recuperados }\end{array}$ \\
\hline SciELO & $\begin{array}{lll}\text { 1. } & \text { (*sociología) } & \text { AND } \\
\text { (bibliometria) } & \\
\text { 2. (*bibliometría) } & \text { AND } \\
\text { (campo científico) } & \end{array}$ & $\begin{array}{l}\text { No responder a las } \\
\text { temáticas } \\
\text { Orientación única a } \\
\text { los estudios } \\
\text { bibliométricos }\end{array}$ & $\begin{array}{l}\text { Artículos: } 23 \\
\text { Comunicación } \\
\text { breve: } 1\end{array}$ & Artículos: 6 \\
\hline DOAJ & $\begin{array}{lr}\text { 1. campo científico. } \\
\text { Subject: Social Sciences } \\
\text { 2. bibliometría. Subject: } \\
\text { Bibliography. Library } \\
\text { science. Information } \\
\text { resources }\end{array}$ & $\begin{array}{l}\text { No responder a las } \\
\text { temáticas } \\
\text { Presencia de } \\
\text { duplicados a partir del } \\
\text { solapamiento } \\
\text { No orientarse a las } \\
\text { áreas de sociología o } \\
\text { de bibliotecología y } \\
\begin{array}{l}\text { ciencias de la } \\
\text { información }\end{array}\end{array}$ & Artículos: 51 & Artículos: 8 \\
\hline $\begin{array}{l}\text { Google } \\
\text { Scholar }\end{array}$ & 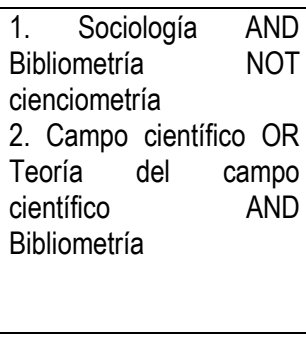 & $\begin{array}{l}\text { Tener una orientación } \\
\text { cienciométrica } \\
\text { No abordad las } \\
\text { relaciones entre } \\
\text { campo científico } \\
\text { Centrarse en } \\
\text { fundamentar aspectos } \\
\text { tecnocráticos de la } \\
\text { bibliometría }\end{array}$ & $\begin{array}{l}\text { Libros: } 27 \\
\text { Artículos: } 62 \\
\text { Ponencias: } 3 \\
\text { Tesis: } 6\end{array}$ & $\begin{array}{l}\text { Libros: } 22 \\
\text { Artículos: } 9 \\
\text { Ponencias: } 1 \\
\text { Tesis: } 2\end{array}$ \\
\hline
\end{tabular}




\begin{tabular}{|l|l|c|c|c|}
\hline & $\begin{array}{l}\text { 3. Evaluación de la } \\
\text { ciencia AND Campo } \\
\text { científico }\end{array}$ & & & \\
\hline Total & -- & -- & 173 & 48 \\
\hline
\end{tabular}

Fuente: Elaboración propia.

Lo anterior fue estandarizado a fin de realizar valoraciones sobre los autores con mayor autoridad en las temáticas y las relaciones entre las temáticas que aborda el estudio. Finalmente se obtienen 48 documentos relevantes recuperados que fueron citados en el presente estudio. Se definieron dos categorías y varias unidades para la orientación del estudio documental:

1) Revisión, análisis y recuperación de la sociología del conocimiento y la Teoría de los Campos

- La sociología del conocimiento en el análisis de campos científicos

- La Teoría de los Campos de Pierre Bourdieu y su contexto en la ciencia

- Las comunidades científicas y su análisis

2) Identificación de nexos entre los aspectos mencionados anteriormente:

- Principales antecedentes que desde la sociología del conocimiento emplean el método bibliométrico

- Los estudios métricos y la bibliometría para delimitar la estructura campos científicos

- Capitales y su relación con los indicadores bibliométricos

\section{Estructura y configuración del campo científico}

La lógica de campo implica el pensamiento racional en tanto las relaciones son las que delimitan el mundo social. De esta forma, el campo es definido por Bourdieu como "un espacio estructurado de posiciones, como una red de relaciones objetivas (de dominación, de subordinación, de complementariedad, entre otras) entre posiciones" (Schleifer, 2008, p. 237). A criterio de Wacquant (2004), la Teoría de los Campos se aplica tanto en la construcción de conocimiento como en la praxis de la ciencia. La concepción de Bourdieu en relación con la conformación del campo científico, radica en el sistema de relaciones objetivas.

Es el lugar (...) de una lucha de concurrencia, que tiene por apuesta específica

el monopolio de la autoridad científica, inseparablemente definida como capacidad técnica y como poder social, 0 , si se prefiere, el monopolio de la competencia científica, entendida en el sentido de capacidad de hablar y de actuar legítimamente (es decir, de manera autorizada y con autoridad) en materia de ciencia, que está socialmente reconocida a un agente determinado (Bordieu, 1976, p. 12).

Bourdieu enuncia que el sistema de funcionamiento de los campos gira alrededor de los capitales y su apropiación. La Teoría de los Campos de Bourdieu tiene entre sus principios el de la configuración de las prácticas de los agentes en torno a las estructuras de relaciones objetivas (Bourdieu, 2003).

Los campos científicos se constituyen a partir de la lucha científica y los actores que intervienen en los procesos de la actividad y producción de conocimientos, dando lugar a estados antagónicos. 
Puesto que todas las prácticas se orientan hacia la adquisición de la autoridad científica (prestigio, reconocimiento, celebridad, etc.), búsqueda intrínsecamente doble, lo que llamamos comúnmente "interés" por una actividad científica (una disciplina, un sector de esta disciplina, un método, etc.) tiene siempre dos caras; y lo mismo ocurre con las estrategias que tienden a asegurar la satisfacción de este interés (Bourdieu, 1994, p. 133).

Al respecto, se evidencian dos manifestaciones dentro de los campos: 1) la dominante o conservadora orientada a mantener el estado de la estructura establecida y 2) la emergente que tiene como propósito el de subvertir la distribución de los capitales.

Así, la definición de la cuestión de la lucha científica forma parte de las posiciones en la lucha científica, y los dominantes son aquellos que consiguen imponer la definición de la ciencia según la cual su realización más acabada consiste en tener, ser y hacer lo que ellos tienen, son o hacen (Bourdieu, 1994,

p. 137).

El campo científico como espacio de lucha por la autoridad en la ciencia, le atribuye a cada agente, en dependencia de su lugar dentro de la estructura, problemáticas de carácter científico y político. De lo anterior se sustenta que la asunción de estrategias, paradigmas, perspectivas y métodos en la ciencia; se corresponden con estrategias políticas con una visible correspondencia en materia de ciencia.

La concepción de campo científico implica poner la competencia en función de la apropiación de los capitales. Bourdieu, Chamboredon y Passerón (2002), establecen que la sociología del conocimiento tiene por objetivo valorar y examinar las condicionantes sociales al que deben someterse los agentes para entrar en el "juego" del campo. La producción científica como prácticas objetivadas constituye la parte visible de los campos a través de los documentos generados. A criterio de Moody (2004), "los estudios en sociología del conocimiento han sugerido que la producción de un núcleo de ideas generadas por los científicos, están en función del grupo de personas con las que interactúan" (p. 213).

En el campo fluctúa un capital científico que lo empodera simbólicamente. En el análisis de la configuración del campo de la comunicación política en México a partir de los documentos publicados, Rodríguez (2017), establece que fundamentalmente son "cuatro capitales conforman los campos científicos: el capital relacional, el de autoridad, el de producción académica y el económico" (p.17). En la interacción de los investigadores tienen lugar los intercambios, juegos, competencias relativos a los capitales del campo. Respecto a lo anterior Pacheco (2006) establece que:

A todo campo corresponde cierto tipo de instancias y agentes encargados de la producción y reproducción, tanto de prácticas y procesos particulares como de saberes, habilidades, conocimientos y formas de representación simbólica que lo distinguen de otros campos (...). Un campo como el científico radica en su capacidad para incorporar y producir normas y valores que regulen socialmente el conjunto de procesos y productos de orden científico (p. 98).

En el campo científico se identifican dos grandes grupos: los dominantes y los dominados. Los dominantes cuentan con el monopolio de definición de la ciencia. Son autoridad conocida y reconocida, por lo que pelearán por conservar el estado de las relaciones de fuerza. Los sectores dominantes no ejercen su condición directamente sobre los individuos sino sobre 
el campo, estableciendo pautas, diseñando métodos y proponiendo lo que es y lo que debe ser ciencia. Los dominados o los ingresantes tienden a ocupar, a los ojos de Bourdieu, el rol de herejes o de subversivos ya que intentarán modificar las relaciones de fuerza internas (Schleifer, 2008). Los sectores minoritarios deben desarrollar estrategias de ruptura y que a la vez les permitan permanecer dentro del campo. Los agentes sociales tienen y mantienen intereses particulares y colectivos en el juego, según sus disposiciones, según la posición que ocupen y según la estructura y el volumen del capital.

\section{Sociología del conocimiento y análisis de la ciencia}

Para el análisis de campos científicos, desde la óptica de la sociología del conocimiento, es necesario reconocer las teorías que han puesto a la ciencia como objeto de estudio. Se trata de un conjunto de saberes que no solo se limita al ámbito de la sociología del conocimiento, sino que transgrede otras disciplinas como la sociología de la ciencia y la sociología del conocimiento científico. La ciencia como producto social tiene lugar a través de la actividad y producción científica. La actividad científica se configura como las acciones y profesiones que tienen como objetivo generar ciencia. La producción científica es la parte materializada de dicha actividad y se expresa en documentos, generalmente publicados.

El objeto de estudio de la sociología del conocimiento tiene una orientación a identificar la producción de conocimientos donde por el contexto, la ideología, las condiciones económicas y políticas; son determinantes. El conocimiento como producto cultural a criterio de Mannheim (1987), es puede ser comprendido desde tres planos: el plano objetivo inmediato (el conocimiento como dato autónomo), el plano expresivo - subjetivo y el plano documental. Este último plano que a decir de Gómez (1993), "se manifiesta a través de y en indisoluble unidad con los anteriores" (p. 49), es el que refiere a los medios donde se materializa el conocimiento, la fuente, el documento. El plano documental también alude a la presencia de una cosmovisión de carácter social y solo es abordado con éxito desde una sociología del conocimiento.

El análisis de campos científicos incluye la determinación social del conocimiento. Las ideas no tienen una existencia independiente, sino que siempre se concretizan en condiciones socio - económicas específicas. Los aspectos mencionados permiten caracterizar las políticas y los medios de financiamiento que sostiene la investigación en campos científicos particulares. El análisis de la infraestructura científica permite identificar los medios de producción donde la institucionalización y las publicaciones juegan un papel protagónico.

Berger y Lukman (1976), establecen que la construcción del conocimiento es reflejo de la sociedad, por lo que a partir de la ingeniería inversa: analizando los documentos, se puede caracterizar un momento histórico concreto. Al respecto, el autor Rizo (2015) aborda el método de lectura atenta de los textos que se producen como medidor de la sociedad. Desde el análisis de la sociología del conocimiento se vinculan las causas del origen del conocimiento en relación con la comunidad científica que la produce. La disciplina estudia estas determinaciones: las relaciones recíprocas entre la estructura social y la estructura cognoscitiva, la forma en que la conciencia individual se transforma en producto científico, fruto de la confluencia de determinantes sociales que envuelven al individuo y lo anteceden. Lo anterior permite caracterizar a las comunidades de práctica que configuran la estructura de los campos científicos. 
Bourdieu, Chamboredon y Passerón (2002), mencionan el elemento subjetivo en la ciencia al establecer "la objetividad de la ciencia no podría descansar en un fundamento tan incierto como la objetividad de los científicos" (p. 374). Bourdieu plantea una teoría del conocimiento sociológico donde establece las relaciones entre teoría - metodología, teoría práctica, sujeto - estructura donde se integran las concepciones de capital cultural, simbólico, económico, habitus, práctica y estrategia. El concepto de habitus refiere las normas y comportamientos de los sujetos en torno a la estructura del campo. Lo anterior a criterio Corrales (2016), se traduce como lógica de significación.

Bourdieu, Chamboredon y Passerón (2002), establecen que la sociología del conocimiento tiene como bjbeto fundamental el de examinar y contrastar las condicionantes a los que son sometidos los científicos, además de aportar a validar ese conocimiento. Dentro de las disciplinas que permiten obtener herramientas para analizar y describir campos científicos se encuentra la sociología de la ciencia. Entre otros aspectos, se encarga de identificar las formas en que se organiza la comunidad científica y sus discursos científicos. Resulta relevante para esta disciplina el análisis de la producción y difusión de la ciencia, aspecto que permite develar prácticas objetivas y subjetivadas dentro de los campos.

La configuración del ethos (de la ciencia) de Robert K. Merton emite una serie de planteamientos en el ámbito normativo de las prácticas de las instituciones. El ethos constituye el universalismo, comunalismo, desinterés y escepticismo organizado (Torres, 2013). Contiene las normas tanto éticas como morales que son compartidas por los miembros de una comunidad científica. Merton describe la labor científica a partir del establecimiento de una serie de metas, técnicas y valores que deben compartir los investigadores. La práctica de la sociología del conocimiento científico incluye el estudio de campos científicos y la explicación de los puntos de contingencia o flexibilidad interpretativa, donde existen ambigüedades. Tales variaciones están relacionadas con una serie de factores políticos, históricos, culturales o económicos. El programa fuerte de la sociología del conocimiento científico atribuido a David Bloor, pretende "ofrecer una explicación social del producto de la actividad científica" (Márquez y Vilaró, 2014, p. 79). Los productos de la ciencia se traducen fundamentalmente en documentos que reflejan los hallazgos del conocimiento. El programa estudia las formas en que influye la estructura social en los diferentes objetos de investigación. El programa recibió numerosas críticas por varios científicos al catalogarlo esencialmente como ambivalente. Los principios en los que se sustenta el programa fuerte de la sociología del conocimiento científico son recogidos por Bloor (1994, 1998), estos son:

1. Causalidad. Ocuparse de las condiciones que dan lugar a las creencias o a los estados de conocimiento.

2. Imparcialidad. Con respecto a la verdad y la falsedad, la racionalidad y la irracionalidad, el éxito o el fracaso.

3. Simetría. Los mismos tipos de causas deben explicar, las creencias falsas y las verdaderas.

4. Reflexividad. sus patrones deberían ser aplicables a la sociología misma. Como el requisito de simetría, ésta es una respuesta a la necesidad de buscar explicaciones generales.

El principio de la reflexividad permite fundamentar las expresiones de las prácticas objetivadas en campos científicos. Bourdieu (2003) establece que la reflexividad en el ámbito 
científico tiene una doble función: 1) identificar la realidad en la que se contextualiza y 2) tomar una "posición integradora que consiste en poner especialmente entre paréntesis aquello que las teorías confrontadas pueden deber a la búsqueda ficticia de la diferencia: lo mejor que se puede sacar de una historia de los conflictos" (p. 21).

El principio de la reflexividad se presenta como una herramienta sociológica con visión autocrítica al servicio de la colectividad, para que, a su vez; entre en un proceso reflexivo. Se hace conciencia en las representaciones que se tiene de las acciones considerando las implicaciones sobre las propias acciones, lo que lleva a nuevas representaciones que involucran cambio, novedad y dinamismo (Ferreira, 2005). El autor citado la presenta como el autoajuste en el quehacer científico, permitiendo una ciencia activa debido a que "sólo mediante el conocimiento de nuestro conocimiento y la experimentación sobre nuestra experiencia podremos acceder a las realidades constitutivas del mundo" (p. 6).

En correspondencia Luhmann (1991), establece que "la reflexividad se realiza, necesariamente, como comunicación de la comunicación" (p. 44). En adición, para poder comprender las interacciones Ferreira (2005), apunta "la ciencia de lo social se constituye como tal en virtud de su propia socialidad" (p. 8). La reflexividad se plantea como acción de abrir la caja negra de la investigación y las teorías utilizadas. A criterio de Rodríguez (2017), permite explorar los sistemas subjetivos de predisposiciones y expectativas: sus habitus estructurantes del campo. La descripción del comportamiento de campos científicos contempla las prácticas objetivadas que se manifiestan en la producción científica.

\section{Bibliometría y capitales en juego en la configuración de los campos científicos}

Para la descripción de un campo científico es necesaria la identificación de capitales particulares que se manifiestan en su interior. Muchos de los capitales en juego se generan en la objetivación de la actividad académica que se manifiesta en documentos. Los capitales más comunes dentro de la Teoría de los Campos son: cultural, económico, social y simbólico. El capital simbólico está integrado por cualquier otro tipo de capital en función de legitimidad. Es la forma que los distintos tipos de capital (económico, relacional, cultural, social, entre otros) adoptan cuando son conocidos y reconocidos por los otros agentes sociales. Se traduce en conocimiento, consagración y legitimidad. Forma parte de los elementos estructurantes como consecuencia de las decisiones que toman los agentes que dan una forma particular al campo a través de la "construcción social de la ciencia y de procesos como el establecimiento de agendas de investigación y docencia, de temáticas de congresos, priorización de temas para becas y otras materializaciones del mismo estilo" (Rodríguez, 2017, p. 21).

El capital simbólico y científico se encuentran estrechamente ligados a los procesos de legitimación del conocimiento y lo que se comunica de este. La posición depende a su vez del capital simbólico institucionalizado que impulsa a conservar o subvertir el estado del campo. Las exigencias del capital simbólico se refuerzan en la configuración de los campos. Desde la perspectiva de Williams (1980), acerca de lo dominante, emergente y residual; siendo predominante el enfoque dominante en correspondencia con la asimilación de la competencia en función de los capitales que establece la lucha por la autoridad científica. Este enfoque privilegia las producciones discursivas que procuran mantener la tradición investigativa en términos de paradigmas, siendo hegemónico y terminando siendo aceptado y asimilado por los agentes. El enfoque emergente tiene en su contra a toda la lógica del sistema que, desde la 
acción de los árbitros mediante su capital simbólico, censuran toda forma de manifestación de este enfoque; por lo que la producción intelectual de los sujetos suele ser escasa y no se refleja en el principal medio de socialización de la ciencia: las revistas científicas.

Desde las relaciones entre bibliometría y Teoría de los Campos Científicos algunos de los capitales identificables son:

- La búsqueda de autoridad científica (capital de autoridad)

- Capital relacional (establecimiento de redes y asociaciones de un investigador)

- Capital de producción académica

- La citación como capital científico ${ }^{1}$

Además de identificar las relaciones entre científicos en torno a los capitales en juego, existen otros aspectos para caracterizar un campo científico en particular. Crane (1972), aborda indicadores de organización social entre los que se encuentran: "discusiones informales de investigación, publicaciones en colaboración, relación con maestros y la influencia de colegas en la selección de problemas y técnicas de investigación" (p.41). Los aspectos anteriores son manifestaciones de una ciencia consolidada² (Knorr, 2005).

Al respecto, Bourdieu (2005) establece que para analizar la estructura del campo se deben seguir los siguientes aspectos:

1. Situar al campo en cuestión en el espacio social global del poder y al mismo tiempo, analizar su evolución en el tiempo.

2. Análisis de la estructura interna del campo: relaciones objetivas entre las posiciones objetivas que ocupan los agentes y los grupos que juegan el juego dentro de él (manifestación de la lucha y el juego entre los intelectuales ortodoxos y los subversivos).

3. Análisis de los habitus de los ocupantes de esas posiciones.

El investigador Ribes-Iñesta (2015), establece otros aspectos como: 1) la esencia del campo, 2) la calidad del diseño de investigación y análisis (en términos de diversidad metodológica y distinción interdisciplinar y disciplinar), 3) lo teórico versus la orientación aplicada de la investigación, 4) la infraestructura de la disciplina (eventos, asociaciones e instituciones) y 5) la diversidad de investigadores en el campo.

La lucha por la obtención de capitales es constitutiva de los campos y su habitus. A criterio de Bourdieu (1994), la lucha por la autoridad científica constituye el enfrentamiento constante entre los sujetos de un mismo campo que son sus propios competidores. En consecuencia, la autoridad recae en los sabios, autorizados por sus méritos a evaluar lo verdadero y lo que no lo es. La búsqueda de autoridad científica es un capital acumulable, transmisible y transformable (Bourdieu, 1976). El capital científico se manifiesta en el reconocimiento de los productos de la ciencia. El proceso se genera por un sistema al estilo meritocrático donde se obtienen credenciales para ganar esta autoridad, un reconocimiento socialmente construido y reconstruido (Rodríguez, 2017). "Las interconexiones pueden verse en la manera como el desarrollo del conocimiento es controlado contra las normas profesionales y

\footnotetext{
${ }^{1}$ Se distingue como un capital diferente al de autoridad porque además puede contribuir significativamente a los reconocimientos que puede obtener un investigador a partir del impacto que generan sus producciones intelectuales dentro del campo. Pudiera incidir este capital en el económico y de autoridad a partir de que un investigador altamente citado puede obtener con mayor facilidad que otros un puesto de trabajo 0 un papel destacado en proyectos 0 al frente de instituciones de investigación.

${ }^{2}$ El autor la define como una ciencia hecha o anciana.
} 
la manera comparable de cómo la reputación puede afectar la identificación y la divulgación de las nuevas ideas" (Becher, 2001, p. 94).

La autoridad científica es entonces "hacerse de un nombre" y en dicho proceso los agentes (sujetos o instituciones) deberán contener (aceptar), la lógica y estado del campo, reconociendo los árbitros que les facilitarán su entrada. Dentro de los aspectos que tipifican, por ejemplo, el accionar de los sujetos que desean ingresar a un campo fuertemente autónomo lo constituye la colaboración científica con autores de reconocido prestigio. En muchas ocasiones los sujetos que se inician o que tienen poco capital de autoridad, incluyen en sus producciones académicas a reconocidos profesionales de su área. Lo anterior es denominado como efecto sombra y se revierte en visibilidad para aquellos que cuentan con menor reputación en el ámbito académico, para los agentes con determinado prestigio esto significa continuar aumentando su capital de producción científica; que en muchos casos aumenta por la acción de terceros que por su propia iniciativa.

El capital científico es sobre todo un capital donde el prestigio, la autoridad y el reconocimiento actúan como criterio de medidas. Actúa sobre los intereses del campo, sobre su delimitación de problemas, metodologías y teorías consideradas por su grado de cientificidad (Paláu-Cardona, 2009). Influyen en el aspecto anterior la calidad de los medios de socialización, la jerarquía o rango de las instituciones, la cantidad de proyectos, los fondos obtenidos y su procedencia, los premios y méritos obtenidos y legitimados por el campo (Paz, 2018).

El capital científico está dividido en capital relacional (a partir de redes), de autoridad, de producción académica y económico (Rodríguez, 2017). El capital de producción académica parte de la consolidación del trabajo científico. Se materializa en forma de documentos generalmente publicados en revistas científicas, constituyendo estas el medio de socialización por excelencia; por lo que se obtiene mayor reconocimiento (o capital). Además de las instancias específicamente encargadas de la consagración (academias, premios, entre otras), comprende también los instrumentos de difusión y en particular las revistas científicas que operan en función de los criterios dominantes, consagran los productos conformes con los principios de la ciencia oficial (Bourdieu, 1976). En las revistas se muestra el ejemplo de lo que merece el nombre de ciencia y ejerce censura sobre las producciones heréticas, tanto rechazándolas expresamente, cuanto desanimando simplemente la intención de publicar por medio de la definición de lo publicable que proponen (Bourdieu, 2008). Los enfoques y paradigmas emergentes tienen que ser legitimados por la publicación en revistas, aspecto que rara vez sucede debido a la estrategia de conservación dominante de los campos.

Las revistas científicas como parte de la institucionalización de la ciencia, tienen un papel fundamental. Según Giordanino (2011), juegan diferentes roles en la comunicación científica:

1. Construyen una base colectiva de conocimiento

2. Comunican la información con velocidad y celeridad (además de los preprints y postprints)

3. Validan la calidad de la información (filtran y difunden)

4. Distribuyen recursos (evaluación, registro de invenciones, documentación)

5. Construyen comunidades científicas

El propósito fundamental de las revistas científicas es la publicación de artículos. Esta tipología documental es actualmente el documento científico por excelencia (Repiso, Jiménez - 
Contreras y Aguaded, 2017). La obtención de capital de producción científica se relaciona ineludiblemente con los artículos publicados por los investigadores. La divulgación, evaluación y descripción de la ciencia ha sido un aspecto esencial de la sociología del conocimiento (Cristancho y Posada, 2015; Herrera, 2020). Los documentos científico técnicos publicados son una forma de identificar las prácticas objetivadas de científicos, instituciones y países miembros de un campo.

\section{Relaciones entre indicadores biblométricos y campos científicos}

Una de las formas para identificar los capitales de producción académica dentro de los campos científicos es con el uso de la bibliometría. En 1987 Bruno Latour publicó Science in action. En el texto mencionado acuñó la noción de caja negra —aplicada al análisis social de la CyT- para referirse al desarrollo científico - técnico o de los conocimientos que por su uso han logrado integrarse de forma natural a la cultura científica. El autor establece que paradójicamente, mientras más se generalizan y expanden los dominios y disciplinas del conocimiento; más difuso se tornan estos. Al respecto, la sociología del conocimiento debe "abrir" esas cajas negras y visibilizar el entramado y estructura que las sostiene (GómezMorales, 2015).

Los estudios bibliométricos se enfocan en la evaluación de la actividad científica a través de la literatura científica publicada. Contribuyen a la elaboración de políticas científicas y a criterio de Maltrás (2003) procuran evaluar de forma cuantitativa variables relacionadas con disciplinas, autores, países, regiones y autores. El uso indiscriminado de los estudios bibliométricos ha conllevado a múltiples distorsiones en la caracterización de la actividad y organización de la ciencia. Los indicadores propuestos en la realización de este tipo de investigaciones se corresponden con un modelo de ciencia occidentalizada que bajo la hegemonía de paradigmas establecidos. El origen y propósito de estos indicadores refuerzan las exigencias cognoscitivas de la ciencia globalizada y desprecian los saberes construidos desde otras regiones. Estas lejos de romper con el círculo vicioso al que están sometidos, entran en el juego para buscar legitimidad en el campo donde las reglas impuestas los condenan al fracaso.

La bibliometría permite medir de forma cuantitativa la actividad científica, esta disciplina comprende:

1. Procedimientos estadísticos - matemáticos para identificar tendencias en cuanto al uso, difusión y creación de literatura científica

2. Investigación cuantitativa de repertorios bibliográficos y documentación científico técnica

3. Estudios de usabilidad y alcance de fuentes con carácter científico por parte de una comunidad y sus miembros

4. Análisis de las relaciones objetivas entre investigadores

El análisis de grandes volúmenes de datos y fuentes como vía para delimitar y caracterizar campos, requiere de mucho tiempo por parte de los investigadores. EL volumen de la producción intelectual constituye un indicar de evaluación de los campos. Cada año la producción de conocimientos aumenta de manera proporcional, simétricamente también la cantidad de investigadores y revistas científicas. La recuperación de los documentos debe tomar 
en consideración el solapamiento que a menudo sucede cuando se consultan diversas bases de datos.

Las limitaciones teóricas y metodológicas que se identifican desde los componentes de la teoría: campo, capital y habitus; están determinadas en la selección de las fuentes de información. Aunque las publicaciones son una manifestación de los campos científicos, la selección de solo documentos publicados excluye el resto de la producción científica del campo que no está visible en bases de datos como tesis y libros. Aun cuando un investigador decida recopilar tipologías documentales referentes a un campo en específico que no esté publicada, nunca podrá obtener todas las fuentes producidas por comunidades de científicos. Lo anterior pudiera determinar en que se cuestione si los resultados son verdaderamente reflejo del campo, aspecto que deberá justificarse en la selección de una muestra acorde a los requerimientos de determinados estudios.

El análisis del capital de autoridad, desde la bibliometría, aborda el reconocimiento meritocrático de los agentes del campo. Al respecto, se integran premios, becas de investigación y reconocimientos desde la illusio del científico. Las recompensas disponibles son orientadoras de actitudes y comportamientos de los implicados. EL habitus se mueve en torno al acceso de los reconocimientos que tienen lugar tanto formalmente como informal. El capital de autoridad considera el posicionamiento de instituciones, investigadores y países en rankings. Los principales indicadores bibliométricos asociados al capital relacional son premios obtenidos (por nivel de importancia), cantidad de líderes científicos en líneas y proyectos de investigación, resultados de procesos de evaluación externa como acreditaciones, entre otros.

El capital relacional se constituye por las redes y asociaciones que un investigador construye 0 se suma de acuerdo a sus fines científicos. La descripción del capital relacional muestra la dinámica interna de los campos que se establece entre los sujetos que lo componen. Lo anterior constituye por tanto un reflejo del hábitus de las interacciones entre los sujetos. Los investigadores cuentan con redes formales e informales que se manifiestan en la producción científica. Las principales redes formales que existen son: las asociaciones profesionales, las organizaciones profesionales, los proyectos, grupos e instituciones de investigación y los departamentos académicos. Las redes informales se constituyen de forma espontánea y se identifican desde la perspectiva de los colegios invisibles.

El capital relacional se asocia a la idea de red entre científicos, lo que permite comprender la configuración de un campo. Analiza el "sistema de posiciones y disposiciones sociales que son objetivas y subjetivadas" (Ramírez, 2010, p. 23). Las prácticas subjetivadas se relacionan con la configuración de las redes e integran las leyes o regulaciones del campo. Las redes de intercambio académico parten de las denominadas tribus académicas (Becher, 2001).

La asociación de los agentes del campo es significativa a la hora de producir, socializar y legitimarse dentro de la ciencia. Los campos consolidados presentan bajos índices de transitoriedad a partir de que se evidencian profesionales especializados que publican con regularidad sobre el campo en el que se desenvuelven. Al respecto, son los árbitros los que tienen los medios para apropiarse simbólicamente de la obra científica y para evaluar sus méritos, son los que determinan la legitimidad de los nuevos miembros o de los principiantes que aspiran ingresar al campo.

Las redes académicas permiten la institucionalización de un campo o disciplina cuyas comunidades académicas están agremiadas por diversos intereses. La forma en que se 
configuran y generan patrones de intercambio ayuda a explicar también la manera en que se va constituyendo un campo científico (Rodríguez, 2017). Dentro de los indicadores bibliométricos que permiten analizar el capital relacional, tanto en autores como en elementos dentro del texto se encuentran: 1) el análisis de coocurrencia de términos: en títulos, resúmenes, palabras clave, métodos, técnicas de investigación y elementos citados en las referencias, 2) índice de coautoría, y 3) colaboración entre autores, países e instituciones. Los indicadores relacionados con la coocurrencia de términos permiten además el análisis de "clusters de conocimientos, relaciones de proximidad entre unos y otros nodos, identificar tópicos, textos 0 autores centrales y vínculos entre ellos, así como elaborar mapas de influencias y explorar la evolución de temas científicos" (Ramos, 2012, p. 13).

El capital de producción académica o científica parte de la consolidación del trabajo científico y se materializa en forma de documentos generalmente publicados. El medio de socialización por excelencia del capital mencionado son las revistas científicas. Los enfoques y paradigmas emergentes tienen que ser legitimados por la socialización en el medio mencionado, aspecto que rara vez sucede debido a la estrategia de conservación dominante de los campos. La medición del capital de producción académica desde el método bibliométrico parte de estudios de producción científica. Muestra la distribución del capital a partir del cúmulo de investigaciones realizadas por autores, grupos de investigación, instituciones o países. El volumen de producción da una medida de la consolidación de un campo en particular. La concepción anterior parte de que las ciencias y los campos científicos, se evalúan por el nivel de producción científica. Algunos de los indicadores bibliométicos empleados para describir este capital son: productividad por autores, años, tipología documental, países, instituciones, idiomas, revistas más productivas, índice de transitoriedad, entre otros.

La citación como capital científico se relaciona directamente con el capital de autoridad. Las relaciones entre argumentos son identificables a través de las referencias (Crane, 1972). Las citas legitiman y reconocen la obra de otros investigadores, considerada incluso como la unidad básica del sistema de recompensas (Merton, 1957). En cuanto a la función legitimadora, "permite utilizar el prestigio de alguien con autoridad en el campo para amparar las ideas introducidas, una externalidad que se liga al interior de la dinámica del campo" (Rodríguez, 2017, p. 54).

El análisis relacional entre la productividad científica y los patrones de citación ha sido ampliamente trabajado por Price (1973). La tesis central de este es que la importancia de una teoría científica está expresada por el prestigio de un autor en tanto su volumen de productividad y su nivel de citación (Ramírez, 2010; Vélez, 2010). Al respecto, se genera un campo de atracción a citar a un autor que ha sido más citado que se ha avalado su investigación por terceros. Se evidencia

Las citas como unidad de observación favorecen procesos reflexivos a partir de visualizar las relaciones e influencias entre los agentes del campo. Las citas se convierten en indicadores de conexiones sociales, alianzas y estrategias dentro del sistema de comunicación científica. El análisis de citas como capital científico muestra la estructura relacional y la influencia de unos investigadores sobre otros, por lo que también da una medida del capital de autoridad de los agentes. Los indicadores bibliométricos más empleados son: cantidad de citas por publicaciones y años, estudios altamente citados, autores y revistas más citadas, fuentes más citadas y análisis de coocurrencia de elementos citados. 


\section{Conclusiones}

Los campos científicos se objetivan en la producción científica que, en última instancia, es reflejo del grado de consolidación y desarrollo de un campo en particular. La producción científica constituye una medida de las relaciones que establece la comunidad que se configura a través de prácticas 0 habitus entre los agentes. A partir de los supuestos anteriores se establecen las dinámicas que se legitiman en la actividad científica. Como resultado se generan documentos que por lo general se publican en revistas científicas. El análisis de las publicaciones da una medida del comportamiento de campos científicos a partir de los roles y los autores que intervienen en el dominio y la apropiación del capital.

En consonancia con las afirmaciones anteriores, resulta imprescindible para la sociología del conocimiento la aplicabilidad de un método que le permita procesar y analizar grandes volúmenes de información en torno a la producción intelectual generada. Al respecto, la bibliometría y el método bibliométrico emergen como alternativa orientada al análisis del volumen de la literatura científica. Teoría de los Campos y bibliometría se complementan. El dato cuantitativo y la valoración desde una perspectiva cualitativa que analice las relaciones entre actores del campo, permite la obtención de mejores resultados.

En el campo científico existen cuatro tipos de capitales principales que modulan su estructura: capital de autoridad, relacional, de producción científica y la citación como capital científico. En consecuencia, la existencia de determinados indicadores bibliométricos de producción científica, visibilidad e impacto favorecen a caracterización y delimitación de los campos. La combinación de la perspectiva de la Teorías de los Campos Científicos de Bourdieu con el método bibliométrico, favorece identificar las relaciones y prácticas objetivadas de los agentes. El uso de ambas perspectivas favorece los procesos de reflexividad, necesarios en los estudios sociológicos e informacionales contemporáneos.

\section{Referencias bibliográficas}

Alfaraz, Claudio. (2004). El oficio de científico. Ciencia de la ciencia y reflexividad. Revista Iberoamericana de Ciencia, Tecnología y Sociedad - CTS, 1, (2), España, (Pp. 221225). Extraído de http://www.revistacts.net/contenido/numero-2/el-oficio-de-cientificociencia-de-la-ciencia-y-reflexividad/

Becher, Tony. (2001). Tribus y territorios académicos. La indagación intelectual y las culturas de la disciplina. Gedisa. España.

Berger, Peter y Lukman, Thomas. (1976). La construcción social de la realidad. Amorrortu. Argentina.

Bloor, David. (1994). El Programa Fuerte en la sociología del conocimiento La explicación social del conocimiento. Editorial de la Universidad Nacional Autónoma de México. México.

Bloor, David. (1998). Conocimiento e imaginario social, Hombre y Sociedad. Gedisa. España.

Bourdieu, Pierre. (1976). Le champ scientifique. Actes de la recherche en sciences sociales, 2, (2), Francia, (Pp. 88-104). Extraído de https://www.persee.fr/doc/arss_03355322_1976_num_2_2_3454 
Bourdieu, Pierre. (1990). In other words. Essays towards a reflexive sociology. University Press. Estados Unidos.

Bourdieu, Pierre. (1994). El campo científico. Redes: revista de estudios sociales de la ciencia, 1, (2), Colombia, (Pp. 129-160). Extraído de https://ridaa.unq.edu.ar/handle/20.500.11807/317

Bourdieu, Pierre. (2003). El oficio de científico. Ciencia de la ciencia y reflexividad. Anagrama. España.

Bourdieu, Pierre. (2004). Science of Science and Reflexivity. Polity Press. Estados Unidos.

Bourdieu, Pierre. (2005). Las reglas del arte. Génesis y estructura del campo literario. Anagrama. España.

Bourdieu, Pierre. (2008). Homo academicus. Siglo XXI Editores. Argentina.

Bourdieu, Pierre; Chamboredon, J. C., y Passerón, J. C. (2002). EL oficio del sociólogo. Presupuestos epistemológicos. Siglo XXI Editores. Argentina.

Corrales, Carlos. (2016). La constitución o construcción de sentido. Extraído de https://es.scribd.com/document/348943424/Sobre-la-constitucion-o-construccion-desentido

Crane, Diana. (1972). Invisible Colleges. The University of Chicago Press. Estados Unidos.

Cristancho, Sergio y Posada, Isabel Cristina. (2015). ¿Para quién publicamos? La producción académica de la investigación cualitativa en salud en el contexto de las políticas oficiales y universitarias en ciencia, tecnología e innovación. Revista Facultad Nacional de Salud Pública, 33, (1), España, (Pp. 52-54). Extraído de https://revistas.udea.edu.co/index.php/fnsp/article/view/24535/20779941

Ferreira, Miguel. (2005). La reflexividad social transductiva. La constitución práctico-cognitiva de lo social y de la sociología. Revista Nómadas, 11(1), Colombia, (Pp. 2-17). Extraído de http://revistas.ucm.es/index.php/NOMA/article/view/NOMA0505120287A

Giordanino, Eduardo Pablo. (2011). Las revistas científicas estructura y normalización. Ponencia presentada en el Acuerdo de Bibliotecas Universitarias, Córdoba. Extraído de http://eprints.rclis.org/16059/

Gómez, Juan Carlos. (1993). El retorno de la sociología del conocimiento. De Mannheim a una epistemología de corte weberiano. Reis: Revista española de investigaciones sociológicas, 62, (3), España, (Pp. 45-60). Extraído de https://dialnet.unirioja.es/servlet/articulo?codigo $=766352$

Gómez-Morales, Yuri Jack. (2015). Usos y abusos de la bibliometría. Revista Colombiana de Antropología, 5, (1), Colombia, (Pp.291-307). Extraído de http://www.scielo.org.co/scielo.php?script=sci arttext\&pid=S0486$65252015000100013 \& \operatorname{lng}=$ en\&nrm=iso

Griñan, Dianné y Muñoz, Teresa. (2017a). El campo de los estudios sociales del trabajo en Cuba. Prácticas hacia su institucionalización: la década de los 60. Universidad de La Habana, 283, (2017), Cuba, (Pp. 224-242). Extraído de http://scielo.sld.cu/scielo.php?script=sci arttext\&pid=S025392762017000100017\&lng=es\&nrm=iso

Griñan, Dianné y Muñoz, Teresa. (2017b). Los estudios del trabajo en Cuba. Una sistematización teórico-metodológica. Novedades en Población, 26, (2), Cuba, (Pp. 69-84). Extraído 
de 40782017000200006\&lng=es\&nrm=iso

Herrera, Yisel. (2020). Estudios sociales rurales: campo y producciones científicas. Revista mexicana de sociología, 82, (2), México, (Pp. 281-309). Extraído de http://www.scielo.org.mx/scielo.php?script=sci_arttext\&pid=S018825032020000200281

King, Patricia. (2000). Internalismo, externalismo y autoconocimiento. Revista Hispanoamericana de Filosofía, 32, (96), México, (Pp. 91-100). Extraído de http://critica.filosoficas.unam.mx/index.php/critica/article/view/895

Knorr, Karin. (2005). La fabricación del conocimiento. Editorial de la Universidad Nacional de Quilmes. Argentina.

Luhmann, Niklas. (1991). Sistemas sociales. Alianza Editorial. México.

Maltrás, Barba. (2003). Los indicadores bibliométricos: fundamentos y aplicación al análisis de la ciencia. TREA. España.

Mannheim, Karl. (1987). Ideología y utopía. Introducción a la sociología del conocimiento. Fondo de Cultura Económica. México.

Márquez, Clara y Vilaró, Ignacio. (2014). La resistencia al programa fuerte en la sociología del conocimiento: la asepsia científica y la amenaza del relativismo. Revista Mexicana de Ciencias Políticas y Sociales, 59, (221), México (Pp. 77-98). Extraído de http://www.scielo.org.mx/scielo.php?script=sci_arttext\&pid=S018519182014000200004\&Ing=es\&nrm=iso

Merton, Robert. (1957). The Sociology of Knowledge: Social Theory and Social Structure. The Free Press of Glencoe. Estados Unidos.

Moody, James. (2004). The Structure of a Social Science Collaboration Network: Disciplinary Cohesion from 1963 to 1999. American Sociological Review, 69, (4), Estados Unidos, (Pp. 213-238). https://doi.org/10.1177/000312240406900204

Najman, Jake y Hewitt, Belinda. (2003). The validity of publication and vitation counts for sociology and other selected disciplines. Journal of Sociology, 39, (3), Estados Unidos, (Pp. 62-80). https://doi.org/10.1177/144078330303900106

Pacheco, Teresa. (2006). Aportes de la sociología al estudio de la ciencia como proceso social y producto cultural. Ludus Vitalis, 14, (25), México, (Pp. 95-104). Extraído de https://dialnet.unirioja.es/servlet/articulo?codigo $=2655755$

Paláu-Cardona, Sofía. (2009). Discursos y prácticas en el proceso de estructuración del campo académico de la comunicación en México: los investigadores de la dimensión política de los medios. Tesis de doctorado presentada para optar por el título de Doctor en Estudios Científico Sociales. Universidad de Puebla. México. Extraído de https://rei.iteso.mx/handle/11117/1259

Paz, Luis Ernesto. (2018). Actividad editorial y socialización de la ciencia. Editorial Feijóo. Cuba.

Paz, Luis Ernesto; Núñez, Jorge y Garcés, Roberto. (2019). El análisis de campos científicos a partir de la documentación. Una perspectiva sociológica. Pedagogía y Sociedad, 22, (55), Cuba, (Pp. 295-319). Extraído de https://revistas.uniss.edu.cu/index.php/pedagogia-y-sociedad/article/view/823

Price, Dereck. (1973). Hacia una ciencia de la ciencia. Editorial Ariel. España. 
Ramírez, Liliana. (2010). Campo científico y redes de coautoría en la psiquiatría. La producción científica psiquiátrica mexicana sobre el trastorno de la personalidad. REDES Revista hispana para el análisis de redes sociales, 19(2), España, (Pp. 20-39). https://doi.org/10.5565/rev/redes.255

Ramos, Claudio. (2012). Estructuras de comunicación en el campo de la ciencia social en Chile: un Análisis de redes. REDES Revista hispana para el análisis de redes sociales, 1 , (23), España, (Pp. 7-42). https://doi.org/10.5565/rev/redes.438

Repiso, Rafael; Jiménez - Contreras, Evaristo y Aguaded, Ignacio. (2017). Revistas Iberoamericanas de Educación en SciELO Citation Index y Emerging Source Citation Index. Revista Española de Documentación Científica, 40(4), España, (Pp. 1-13). http://dx.doi.org/10.3989/redc.2017.4.1445

Ribes-Iñesta, Emilio. (2015). ¿Hermenéutica o heurística? Teoría de campo y conceptos aristotélicos: Respuesta a Ricardo Pérez-Almonacid. Acta Comportamentalia, 23, (1), Brasil, (Pp. 47-54). Extraído de http://pepsic.bvsalud.org/scielo.php?script=sci_arttext\&pid=S018881452015000100005\&lng=pt\&nrm=iso

Rizo, Marta. (2015). Construcción de la realidad, Comunicación y vida cotidiana - Una aproximación a la obra de Thomas Luckmann. Intercom - RBCC, 38, (2), Brasil, (Pp. 19-38). https://doi.org/10.1590/1809-5844201522

Rodríguez, Alejandra. (2017). Configuración del campo de la comunicación política: prácticas y redes de investigadores mexicanos. Instituto de Ciencias de Gobierno y Desarrollo Estratégico. México.

Schleifer, Pablo. (2008). Campo científico, ciencia y uso político de la ciencia en el pensamiento de Bourdieu. Revista de la Facultad de Ciencias, 14, (2008), México, (Pp. 227-252). Extraído

https://www.google.com/url?sa=t\&rct=i\&q=\&esrc=s\&source=web\&cd=\&cad=ria\&uact =8\&ved=2ahUKEwjK4LqooMjwAhVOd6wKHQASCxEQFjAAegQIAxAD\&url=https $\% 3$ A\%2F\%2Ffadeweb.uncoma.edu.ar\%2Fviejo $\% 2$ Fmedios $\% 2$ Frevista $\% 2$ Frevista $14 \% 2$ F15-Schleifer.pdf\&usg=AOvVaw3pPyke0Qia9kCuEbpU_HKI

Torres, Carlos. (2013). La sociología del conocimiento y de la ciencia de Robert Merton. Editorial Félix Varela. Cuba.

Vélez, Gabriel. (2010). Las redes de sentido de las redes sociales: Un estudio cienciométrico. Tesis presentada para optar por el grado científico de doctor en Ciencias Sociales y Políticas, Universidad Iberoamericana, Argentina. http://dx.doi.org/10.13140/RG.2.1.1225.4968

Wacquant, Loïc. (2004). Following Pierre Bourdieu into the field. Sociological Review, 5, (4), Estados Unidos, (Pp. 387-414). https://doi.org/10.1177/1466138104052259

Williams, Raimond. (1980). Teoría cultural Marxismo y literatura. Península. España. 